\title{
Neisseria Meningitidis Urethritis in a Thai Male
}

\author{
Jaray Tongtoyai, M.Sc. ${ }^{1,2}$, Nongkran Tatakham, M.Sc. ${ }^{1,2}$, Thitima Cherdtrakulkiat, M.Sc. ${ }^{1,2}$, \\ Pachara Sirivongrangson, M.D. ${ }^{3}$, Andrew Hickey, Ph.D. ${ }^{1,2}$
}

'Division of HIV/AIDS Prevention, U.S. Centers for Disease Control and Prevention, Atlanta, Georgia 30333, The United Stated Of America.

${ }^{2}$ Thailand Ministry of Public Health - U.S. Centers for Disease Control and Prevention Collaboration, Mueang, Nonthaburi 11000, Thailand.

${ }^{3}$ Department of Disease Control, Thailand Ministry of Public Health, Mueang, Nonthaburi 11000, Thailand.

Received 21 May 2020 • Revised 1 July 2020 • Accepted 12 July 2020 • Published online 16 November 2020

\begin{abstract}
:
Although Neisseria meningitidis (N. meningitidis) urogenital infections have been reported widely, meningococcal urethritis has not been reported previously in Thailand. A 42-year-old Thai male presented at a sexual health clinic with dysuria and urethral discharge following oral and insertive anal intercourse. N. meningitidis, serogroup C was cultured from a urethral discharge specimen and the patient was treated successfully with standard treatment for gonococcal urethritis. This case reflects a growing trend of reports describing meningococcal urethritis, likely resulting from sexual contact.
\end{abstract}

Keywords: meningococcal urethritis, men who have sex with men, Neisseria meningitidis, urethritis

Contact: Andrew Hickey, Ph.D.

Thailand Ministry of Public Health - U.S. Centers for Disease Control and Prevention Collaboration, Mueang, Nonthaburi 11000, Thailand.

E-mail: anh9@cdc.gov

This is an open access article under the CC BY-NC-ND license

(http://www.jhsmr.org/index.php/jhsmr/about/editorialPolicies\#openAccessPolicy). 


\section{Introduction}

Neisseria meningitidis ( $N$. meningitidis) is an invasive bacterial pathogen most notably associated with severe systemic illness with inflammation of the meninges, septicemia, and high mortality. ${ }^{1}$ More than one million infections are estimated each year, many of these occurring in the African meningitis belt, most commonly occurring among infants and adolescents/young adults. In addition, some laboratorians may be at increased risk for contracting $N$. meningitidis infections resulting from infectious aerosols generated during laboratory procedures.

Recently, meningococcal urogenital infection has been reported as a growing issue worldwide. Clusters of meningococcal illness have been noted among men who have sex with men (MSM) in the United States (U.S.), Japan, and elsewhere. ${ }^{2-7}$ In the U.S., serogroup C was responsible for approximately $85.0 \%$ among MSM and only $26.0 \%$ among non-MSM of all serotyped $N$. meningitidis infections. ${ }^{8}$ Bacteremia (45.0\%) and meningitis (50.0\%) were the most common meningococcal disease presentations among MSM, with other clinical presentations such as urethritis still relatively rare. Although meningococcal urethritis has not been reported in Thailand previously, Thailand could see the emergence of local meningococcal outbreaks. The risk is further complicated by the continued high burden of human immunodeficiency viruses (HIV), which has been associated with increased risk of meningococcal infection among MSM.

Colonization of the nasopharynx by $N$. meningitidis increases the risk of urethral infections for sexual partners resulting from unprotected oral sex. Additional risky behaviors, such as recreational drug use and prior history of gonorrhea infection(s), have also been associated with urethral meningococcal infections. ${ }^{2,3}$

Meningococcal urethritis presents with gram-negative intracellular diplococci (GNID), with or without gram-negative extracellular diplococci (GNED), and polymorphonuclear leukocytes (PMNs), making it difficult to distinguish from gonococcal urethritis. As a result, the burden of urogenital infections caused by $N$. meningitidis is likely underappreciated.

We describe the first case of meningococcal urethritis in Bangkok, Thailand, identified as part of the Enhanced Gonococcal Antimicrobial Surveillance Programme (EGASP).

\section{Case report}

In November 2015, Thailand began surveillance for antimicrobial resistant Neisseria gonorrhoeae ( $N$. gonorrhoeae) through the EGASP. On April 26, 2017, a 42-year-old HIV-negative Thai male presented to the clinic with complaints of 5 days of dysuria and purulent urethral discharge. His symptoms developed two days after unprotected receptive oral and insertive anal intercourse with a male partner. He had had a new sexual partner for the past month, and the partnership ended prior to the presentation of these symptoms; the partner could not be contacted. His patient history was notable for presentation at the clinic twice before, in July 2016 and November 2016, with similar complaints as well as one additional time after this episode, in January 2018 (Table 1). Prior to each episode of urethritis, the patient reported participating in group sex, having insertive anal intercourse without a condom, and sex with men and women.

Gram-stain and culture were performed from urethral swab samples collected during the April 2017 visit. A large number (>40 cells/oil power field) of PMNs with GNED and GNID (Figure 1) were observed, consistent with gonococcal urethritis. The patient was given standard treatment for presumptive gonococcal urethritis with 250 milligrams (mg) ceftriaxone administered intramuscularly, and four tablets of $250 \mathrm{mg}$ azithromycin provided orally as a single dose according to the Thai national guidelines. ${ }^{9}$ 
Table 1 Case report history of Neisseria meningitidis urethritis and clinic visits

\begin{tabular}{lllll}
\hline \multirow{2}{*}{ Items } & \multicolumn{3}{c}{ Visit } \\
\cline { 2 - 5 } & $\mathbf{1}^{\text {st. }: \text { JUL } 2016}$ & $2^{\text {nd. }}:$ NOV 2016 & $3^{\text {rd }}:$ APR 2017 & $4^{\text {th. }: \text { JAN 2018 }}$ \\
\hline Gram stain for GNID & Positive & Negative & Positive & Positive \\
Presumptive diagnosis & Gonorrhea & NGU & Gonorrhea & Gonorrhea \\
Culture for N. gonorrhoeae & N. gonorrhoeae & Not found & N. meningitidis & N. gonorrhoeae \\
\hline
\end{tabular}

GNID=gram-negative intracellular diplococci, NGU=non-gonococcal urethritis, JUL=July, NOV=November APR=April, JAN=January, N. gonorrhoeae=Neisseria gonorrhoeae

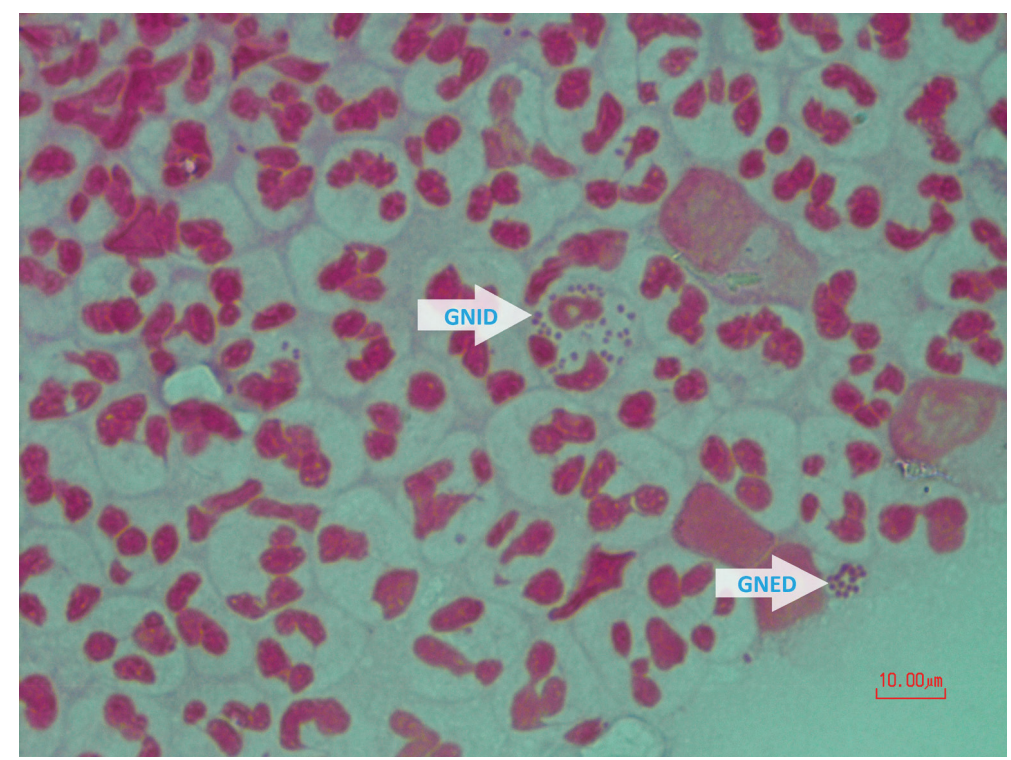

Figure 1 Gram-negative intracellular diplococci and gram-negative extracellular diplococci (arrows) as they appeared on Gram stain

The patient's symptoms resolved within four days and he was advised to notify his partner for treatment. No further follow up testing was performed.

A urethral specimen from the patient was plated on modified Thayer-Martin agar and incubated at 35$36.5{ }^{\circ} \mathrm{C}$ in a $5.0 \% \mathrm{CO}_{2}$ incubator for up to 48 hours. Typical Neisseria colonies were observed after 24 hours and were further characterized by presumptive tests and identified by carbohydrate utilization. Additional investigations using traditional and molecular techniques verified the cultured isolate as $N$. meningitidis, serogroup $C$. Antimicrobial susceptibility testing (disk diffusion: BD SensiDisc $^{\mathrm{TM}}$ ) indicated the isolate was broadly susceptible to the tested antibiotics (cefotaxime, ceftriaxone, meropenem, trimethoprim-sulfamethoxazole, chloramphenicol, and ciprofloxacin). Minimal inhibitory concentrations (determined by Etest (bioMerieux)) were 0.04 micrograms/milliliter $(\mu \mathrm{g} / \mathrm{mL})$ ceftriaxone, $0.016 \mu \mathrm{g} / \mathrm{mL}$ cefixime, $0.50 \mu \mathrm{g} / \mathrm{mL}$ azithromycin, $4 \mu \mathrm{g} / \mathrm{mL}$ gentamicin, and $0.004 \mu \mathrm{g} / \mathrm{mL}$ ciprofloxacin. 


\section{Discussion}

This is the first reported case of $N$. meningitidis urethritis from Thailand and supports the growing compilation of case reports describing meningococcal urethritis among men. ${ }^{2,3}$ As the frequency of meningococcal urethritis increases, healthcare providers may consider $N$. meningitidis, in additional to $N$. gonorrhoeae, as part of the differential diagnosis for symptomatic urethritis. ${ }^{10}$ Although standard antibiotic therapy provided for syndromic management of gonorrhea infection is usually effective for meningococcal urethritis, as in this case, N. meningitidis urethritis could contribute to the development of antimicrobial-resistant gonococcal urethritis as a result of co-colonization and exchange of genetic determinants between the two Neisseria species. ${ }^{11}$

Urethral meningococcal infections can be transmitted during receptive oral intercourse $e^{4,6}$ or condomless insertive anal intercourse with a partner with meningococcal infection. $^{12}$ This patient reported unprotected receptive oral intercourse and condomless insertive anal sex preceding his urethral symptoms and had repeated episodes of gonococcal urethritis before and after the meningococcal urogenital infection. As a result of only limited patient behavioral information being collected as part of the EGASP surveillance program, although the meningococcal infection likely occurred following sexual contact, there was not sufficient behavioral data for the patient and his sexual partners to definitively establish the route of infection.

Guidelines recommend that sexually active MSM should be screened annually for $N$. gonorrhoeae infection. ${ }^{13}$ Nucleic acid amplification tests are widely used for $N$. gonorrhoeae screening at different anatomical sites, although these tests are not able to detect $N$. meningitidis ${ }^{5}$ and culture is necessary to identify meningococcal urethritis. ${ }^{12}$ Guidelines recommend that $N$. meningitidis testing be done in a biological safety cabinet with appropriate personal protective equipment according to biosafety level 2 standard practices. ${ }^{14}$ Routine culture processes can generate infectious aerosols of $N$. meningitidis, which can be a significant occupational risk to laboratory personnel without the proper workplace biosafety controls. ${ }^{14}$ Settings that culture for $N$. gonorrhoeae may not be aware of the potential for $N$. meningitidis urethral infection, and may consider control steps to protect the safety of laboratory staff.

The Advisory Committee on Immunization Practices recommends routine meningococcal vaccination for adolescents and persons at increased risk for meningococcal disease. Although the recommendations do not specifically identify MSM as a high-risk group, increase frequency of meningococcal urethritis globally may point to specific risk behaviors that could be associated with epidemics. A targeted community-based meningococcal vaccination program was used to interrupt an outbreak of meningitis among MSM in New York City, U.S. following prompt implementation of a vaccination program. ${ }^{15}$ Early recognition of meningococcal urethritis events depends on astute clinical recognition of the pathogen and accurate surveillance data on the prevalence, incidence, and risk factor information for meningococcal as well as other causes of urethritis. In addition, meningococcal vaccinations could supplement occupational health programs for laboratory workers that could be exposed to potential aerosols of $N$. meningitidis resulting from routine laboratory procedures, particularly in hyperendemic and epidemic regions.

\section{Conclusion}

The case reflects a growing trend of reports describing meningococcal urethritis, likely resulting from sexual contact. Healthcare providers and laboratory personnel may consider $N$. meningitidis as part of the differential diagnosis for symptomatic urethritis. 


\section{Conflict of interest}

The authors declare no potential conflicts of interest with respect to the research, authorship, and/or publication of this article.

\section{References}

1. van Kessel F, van den Ende C, Oordt-Speets AM, Kyaw MH. Outbreaks of meningococcal meningitis in non-African countries over the last 50 years: a systematic review. J Glob Health 2019;9:010411.

2. Bazan JA, Peterson AS, Kirkcaldy RD, Briere EC, Maierhofe C, Turner AN, et al. Notes from the field: increase in Neisseria meningitidis-associated urethritis among men at two sentinel clinics - Columbus, Ohio, and Oakland County, Michigan, 2015. MMWR Morb Mortal Wkly Rep 2016;65: 550-2.

3. Bazan JA, Turner AN, Kirkcaldy RD, Retchless AC, Kretz $\mathrm{CB}$, Briere $\mathrm{E}$, et al. Large Cluster of Neisseria meningitidis Urethritis in Columbus, Ohio, 2015. Clin Infect Dis 2017;65:92-9.

4. Hay PE, Murphy SM, Chinn RJ. Acute urethritis due to Neisseria meningitidis group A acquired by oro-genital contact: case report. Genitourin Med 1989;65:285-6.

5. Katz AR, Chasnoff R, Komeya A, Lee MV. Neisseria meningitidis urethritis: a case report highlighting clinical similarities to and epidemiological differences from gonococcal urethritis. Sex Transm Dis 2011;38:439-41.

6. Genders RE, Spitaels D, Jansen CL, van den Akker TW, Quin KD. A misleading urethral smear with polymorphonuclear leucocytes and intracellular diplococci; case report of urethritis caused by Neisseria meningitidis. J Med Microbiol 2013;62: 1905-6.
7. Hayakawa K, Itoda I, Shimuta K, Takahashi H, Ohnishi M. Urethritis caused by novel Neisseria meningitidis serogroup W in man who has sex with men, Japan. Emerg Infect Dis 2014;20: 1585-7.

8. Folaranmi TA, Kretz CB, Kamiya H, MacNeil JR, Whaley MJ, Blain $A$, et al. Increased risk for meningococcal disease among men who have sex with men in the United States, 2012-2015. Clin Infect Dis 2017;65:756-63.

9. Bureau of AIDS TB and STIs, Department of Disease Control, Ministry of Public Health of Thailand. Guidelines for the treatment of sexually transmitted diseases 2015. Bangkok: Bangrak STIs Cluster; 2015.

10. Karolus JJ, Gandelman AL, Nolan BA. Urethritis caused by Neisseria meningitidis. J Clin Microbiol 1980;12:284-5.

11. Kahler CM. Emergence of a urogenital pathotype of Neisseria meningitidis. Trends Microbiol 2017;25:510-2.

12. Gutierrez-Fernandez J, Medina V, Hidalgo-Tenorio C, Abad R. Two cases of Neisseria meningitidis proctitis in HIV-positive men who have sex with men. Emerg Infect Dis 2017;23:542-3.

13. Workowski KA, Bolan GA. Sexually transmitted diseases treatment guidelines, 2015. MMWR Recomm Rep 2015;64:1137.

14. Borrow R, Findlow J, Gray S, Taylor S, Kaczmarski E. Safe laboratory handling of Neisseria meningitidis. J Infect 2014;68: 305-12.

15. Kratz MM, Weiss D, Ridpath A, Zucker JR, Geevarughese A, Rakeman J, et al. Community-based outbreak of Neisseria meningitidis serogroup $\mathrm{C}$ infection in men who have sex with men, New York City, New York, USA, 2010-2013. Emerg Infect Dis 2015;21:1379-86. 